\title{
Jean-François Halté : un parcours au service de la didactique du français
}

\section{Anne Leclaire-Halté}

\section{(2) OpenEdition}

1 Journals

\section{Édition électronique}

URL : https://journals.openedition.org/pratiques/1149

DOI : $10.4000 /$ pratiques. 1149

ISSN : 2425-2042

Éditeur

Centre de recherche sur les médiations (CREM)

\section{Édition imprimée}

Date de publication : 15 juin 2008

Pagination : 15-22

\section{Référence électronique}

Anne Leclaire-Halté, " Jean-François Halté : un parcours au service de la didactique du français », Pratiques [En ligne], 137-138 | 2008, mis en ligne le 15 juin 2008, consulté le 07 décembre 2022. URL : http://journals.openedition.org/pratiques/1149; DOI : https://doi.org/10.4000/pratiques.1149 


\title{
Jean-François Halté : un parcours au service de la didactique du français
}

\author{
Anne Leclaire-Halté \\ Université Henri Poincaré Nancy (IUFM de Lorraine) \\ CELTED, EA 3474
}

Jean-François HALTÉ est né le 8 mars 1947 à Amnéville, en Moselle. Il passe son enfance dans la Lorraine sidérurgique, avec quelques incursions, à l'occasion des vacances scolaires, dans le Dauphiné, d'où sa mère est originaire.

Il entre à 1'Ecole Normale de Montignylès-Metz à l'issue de la classe de troisième, passe le baccalauréat; parallèlement à la formation professionnelle qu'il suit en école normale et à ses premières années d'enseignement en tant qu'instituteur, il s'inscrit à l'université. Il obtient un CELG Lettres Histoire, un DUEL de philosophie, une licence de Lettres modernes, une maîtrise de littérature. Il exerce pendant une brève période le

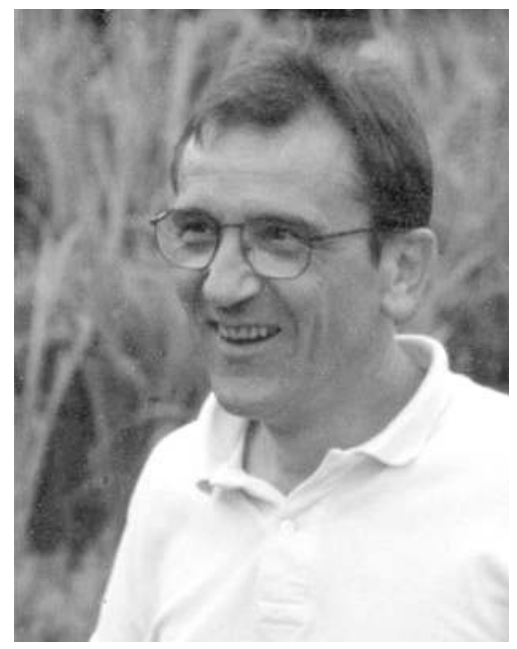
métier d'instituteur (de 1966 à 1968) et enseigne pendant deux ans, au titre de la coopération, à 1'Alliance Française de Cali (Colombie). Il réussit le CAPES de Lettres Modernes, et devient professeur de collège. Il exerce ce métier avec engagement et passion de 1970 à 1982. C'est au début de cette période de sa vie qu'il prend conscience que la meilleure façon de vivre selon ses convictions politiques est de militer contre l'échec scolaire et de revendiquer une école démocratique. Cet engagement est à l'origine de la naissance de Pratiques, revue qu'il fonde en 1974 avec Raymond Michel et André Petitjean. La revue, dont il sera longtemps le co-directeur, est pour lui un outil de recherche et d'expérimentation sur l'enseignement du français. Elle lui permettra d'établir des liens dynamiques entre recherches universitaires à vocation scientifique et problématiques didactiques et pédagogiques. Au sein de la revue, son rôle d'animateur intellectuel s'accompagne d'une réflexion permanente sur la dynamique du groupe dont le collectif bénéficiera longtemps au plan de sa cohésion. 
Après les années d'exercice en collège, fort de son auto-formation sur les questions touchant à l' enseignement du français et de son expérience en matière de formation continue, il est recruté à l'Ecole Normale de Maxéville où il exerce de 1982 à 1988. Il est aussi chargé de cours à l'université de Metz. Il soutient sa thèse en 1985 sous la direction de Jean Peytard (Université de Besançon). Intitulée L'enseignement du français dans le travail en projet, ce travail de 589 pages porte sur l'écriture de fiction et la pratique orale et écrite des discours ordinaires en milieu scolaire. Enseignement-apprentissage de l'écrit d'une part, approche plus globale de l'activité discursive en milieu scolaire d'autre part, ces deux thématiques continueront à traverser les recherches de Jean-François Halté par la suite.

En 1988, il obtient un poste de maître de conférence en Sciences du Langage à Nancy 2. Il soutient son habilitation à diriger des recherches avec une synthèse intitulée Faire de la recherche en didactique du français langue maternelle en 1992, alimentée pour une part importante par la matière du «Que sais-je » sur la didactique du français (1990). Il devient, l'année suivante, professeur à l'université de Metz, où il dispense des enseignements à tous les niveaux, en linguistique générale, en pragmatique, en linguistique textuelle et en didactique. Ses qualités d'enseignant font qu'il est très apprécié de ses étudiants.

Tout ceci n'est qu'une évocation rapide de la vie professionnelle, liée à l'enseignement, de Jean-François Halté. Rendre compte, de façon synthétique, de l'autre versant de cette vie, à savoir les nombreuses activités de formation et de recherche dont a bénéficié la communauté des enseignants et des chercheurs en linguistique et didactique, n'est pas une tâche facile. Elles ont varié au gré des changements de statut et nous essaierons de les évoquer de façon plus ou moins chronologique, autour des grands projets de recherche qui ont jalonné le parcours de Jean-François.

De 1975 à 1988, il a assuré de très nombreux stages de formation continuée, aussi bien pour des enseignants de l'élémentaire, du collège ou du lycée que pour des formateurs ou des formateurs de formateurs, aux niveaux académique et national, dans le cadre des plans académiques de formation ou dans celui des mouvements pédagogiques et autres associations de spécialistes. Il a également assuré la direction d'universités d'été, comme celles intitulées Au cœur de la rénovation des collèges, le français, co-dirigée avec F. Vanoye à St Germain en Laye en 1984, ou Ecrire au collège et au lycée, à Metz en 1987.

Une fois en poste à l'université, Jean-François a continué ses activités de formation, mais sous d'autres formes. Sa spécialisation dans les domaines de la didactique du français l'a amené à effectuer des missions d'expertise, d'animation de séminaires, de formation et d'orientation. C'est ainsi qu'il est intervenu de nombreuses fois à l'étranger, par exemple au Maroc (à Rabat en 1987, à Oujda en 1988, à Meknès en 1992), en Espagne (à Barcelone en 1989), au Liban (à Beyrouth en 1995), au Pérou (Lima en 1997), en Tunisie (1998, 2000). Il a notamment participé, en Suisse, plusieurs années de suite (1997, 1998, 1999, 2000), à une mission visant à mettre en œuvre une réforme de l'enseignement de la culture générale, de la langue et de la communication dans l'enseignement technique.

En termes de recherche, le premier grand chantier ouvert par Jean-François a été consacré au discours explicatif. Responsable d'un groupe de recherche à l'INRP de 1983 à 1989, il a travaillé à l'élaboration d'un modèle du discours explicatif, dans un cadre de type communicationnel, à un moment où il n'existait pratiquement aucune littérature linguistique sur le sujet. Un certain nombre de publications sont liées à cette recherche qui a aussi été à l'origine de quelques thèses que 
Jean-François a dirigées : comptes rendus à l'école élémentaire et formation des compétences explicatives, rôle de l'écriture dans le développement de la compétence explicative, gestion différenciée de 1' « explicatif» dans quelques genres académiques, par exemple.

Le second grand chantier est la réflexion d'ensemble sur la didactique du français qui a conduit à la publication en 1992, dans la collection «Que sais-je?» (PUF), de La didactique du français, ouvrage légèrement remanié pour une réédition en 1993. Au couple dichotomique didactique vs pédagogie, cette réflexion tend à substituer une conception plus intégrative. Pour Jean-François, à l'époque où il écrit ce livre, la didactique du français se partage entre deux courants dominants. Le premier, épistémique, se focalise sur les savoirs à enseigner, les problèmes de leur sélection et de leur transposition dans le champ éducatif. Le second, psychologique (psycholinguistique et psychocognitif), se centre sur les problèmes d'apprentissage inhérents aux objets de savoir. Ils sont issus, plus ou moins directement, des deux branches de la dichotomie ancienne. Jean-François Halté leur préfère en effet une didactique plus intégrative, à vocation finalement praxéologique. La fonction profonde de la réflexion didactique est d'orienter concrètement les pratiques d'enseignement, ce à quoi ne peuvent suffire les deux courants mentionnés. Il suffit de lire les titres des articles de Jean-François postérieurs à La didactique du français pour constater que ce chantier restera en perpétuelle évolution pour lui : de l'élaboration didactique préférée à la notion de transposition, aux questions de configuration du champ, il ne cessera d'affiner sa conception de la didactique du français.

Jean-François s'est également beaucoup intéressé à l'étude des interactions en rapport avec les questions d'apprentissage. En 1993 paraît Inter-actions. L'interaction, actualités de la recherche et enjeux didactiques (collection « Didactique des Textes », université de Metz), ouvrage qu'il dirige et qui réunit des contributions d'A. Trognon, E. Nonnon, R. Bouchard, C. Bachmann, C. Kerbrat-Orecchioni, C. Garcia-Debanc et $\mathrm{P}$. Charaudeau. Le projet consiste à montrer comment et pour quel usage divers secteurs des sciences humaines recourant à la notion d'interaction construisent ce concept. Jean-François introduit l'ensemble de ces contributions par un cadrage général de la problématique théorique et sa mise en relation avec la perspective didactique. Les études concernant l'interaction et l'apprentissage ont dominé son travail les années qui ont suivi cette publication. Il les considère en effet comme un point d'aboutissement et de solidarisation de recherches antérieures dans la mesure où, de proche en proche, c'est l'activité langagière proprement dite, orale et écrite qui s'impose comme thématique capitale. Si linguistique et pragmatique sont les disciplines de référence dominantes, les enjeux pratiques de ces recherches sur le terrain scolaire sont considérables. On peut donner un exemple de ces recherches en renvoyant au numéro 103/104 de Pratiques, «Interactions et apprentissage », paru en 1999, fruit d'un travail collectif et pluricatégoriel qu'il a activement coordonné au sein du CRDF (Centre de recherches en didactique du français), associant université de Metz et IUFM de Lorrraine.

Jean-François a consacré également un certain nombre de ses articles à l'écriture. Lecteur boulimique, amateur en particulier de science-fiction (il a d'ailleurs écrit une nouvelle de science-fiction en 2000), sans doute se serait-il un jour ou l'autre lancé lui-même dans l'aventure de l'écriture fictionnelle si la vie lui en avait laissé le temps. Signe de son intérêt pour l'écriture - il était lui-même un épistolier talentueux -, il a produit plusieurs articles concernant aussi bien 1'orga- 
nisation langagière des discours écrits - produits de l'activité d'écriture - que l'activité de production elle-même, dans ses déterminants cognitifs et sociaux. Son point de vue est que ni l'enseignement des superstructures textuelles, ni la prise en compte des modèles psycholinguistiques des processus rédactionnels, avec leur cécité à la problématique des valeurs (linguistiques, aussi bien que culturelles ou plus largement sociales), ne peuvent suffire à guider la production des textes, soumise d'une part à la variété des genres, entendus comme manière de spécifier les types de textes (un récit comme type de texte peut être spécifié dans un genre comme le roman ou le conte...), et d'autre part, au divers et à l'indéfiniment singulier des sujets producteurs. Il considère qu'écrire relève d'une approche générale de la résolution de problème qu'il convient de préciser, les problèmes d'écriture se caractérisant par le fait qu'ils acceptent une grande variété de solutions. L'un des axes de l'aide que l'enseignant peut apporter à l'élève dans son apprentissage de l'écriture consiste à stimuler ses heuristiques de résolution. L'autre axe est celui des savoirs : ceux-ci, sous leur forme « méta », théorique, gagnent à apparaître pendant ou après l'activité plutôt qu'avant, au moment où, par l'activité elle-même, des structures cognitives d'accueil auront été mises en place. La problématique identifiée classiquement comme celle des passages entre discours écrits et oraux est questionnée et conduit à réarticuler les partitions usuelles en termes de langue et de discours.

Enfin, dans le cadre d'une recherche menée à l'IUFM et initiée en 2002, il s'est intéressé aux problèmes de conceptualisation de l'écrit par les élèves de grande section de maternelle et de cours préparatoire. Malheureusement la maladie a perturbé son engagement dans cette recherche, dont les retombées apparaissent néanmoins dans le dernier texte qu'il a écrit pour les Mélanges du CRAPEL et qui est paru en 2006.

Pour compléter ce portrait professionnel de Jean-François, rappelons sa participation à de nombreux colloques, soit en tant que co-responsable (en particulier les deux colloques de Cerisy (1979 et 1989) consacrés à l'enseignement du français), soit en tant que membre de comités scientifiques d'organisation. Rappelons aussi son appartenance au conseil d'administration de l'Association de Didactique du Français Langue Maternelle (D.F.L.M.). Au niveau local, il a été membre du Conseil scientifique et pédagogique de 1'IUFM de Lorraine. Il a été par ailleurs responsable de la commission «Recherche » de cette institution et a œuvré à ce titre dans le sens d'une collaboration entre les organismes de recherches existants aux plans régional et national.

Enfin, au sein de son université, il a joué un rôle très actif. Il fut directeur du département de sciences du langage (responsabilité à laquelle il a consacré beaucoup d'énergie), de 1994 à 1998, il a été membre du conseil d'UFR et du conseil d'administration de l'université. Il a enfin assumé la charge de directeur du Centre de Recherches en Didactique du Français de l'université de Metz et de l'IUFM de Lorraine et a été responsable du séminaire de didactique du français à l'université de Metz.

Tout au long de son parcours professionnel d'enseignant et de chercheur, JeanFrançois a œuvré pour une école plus juste. Homme de conviction et non de pouvoir, il a consacré toute son énergie intellectuelle à l'avancée de la didactique du français dans le champ des sciences du langage. Il a aussi fait profiter ceux qui ont eu la chance de pouvoir travailler avec lui de la vivacité de son esprit, de sa disponibilité, de sa force chaleureuse et de son humour. 


\section{TRAVAUX, OUVRAGES, ARTICLES}

1974 "Pour une théorie de l'idéologie d'un manuel scolaire », avec A. Petitjean, Pratiques, 1/2, 43-64.

« Essai d'analyse structurale du Chat Noir », avec A. Petitjean et R. Michel, Pratiques, 1/2, 5-28.

«Le code oral : approche théorique », avec A. Petitjean, Pratiques, 1/2, 29-42.

1975 «La grammaire et les textes», Pratiques, 3/4, 45-59.

«Candide : analyse textuelle, pour une application pédagogique »

( $1^{\text {ère }}$ partie), avec R. Michel et A. Petitjean, Pratiques, 3/4, 93-128.

«Candide : analyse textuelle, pour une application pédagogique

( $2^{\text {ème }}$ partie) $)$, avec R. Michel et A. Petitjean, Pratiques, 5, 95-135.

«Candide : analyse textuelle, pour une application pédagogique

( $3^{\text {ème }}$ partie) », avec R. Michel et A. Petitjean, Pratiques, 6, 75-106.

«Le discours de la lettre, la lettre du discours. Analyse d'une lettre de

Victor Hugo », avec R. Michel et A. Petitjean, Pratiques, 6, 13-24.

1976 «L'aiguille creuse : l'enjeu idéologique d'un roman policier », avec R. Michel et A. Petitjean, Pratiques, 11/12, 29-49.

«Le merle blanc: exercice de lecture et d'écriture », avec J.-M. Adam et A. Petitjean, Pratiques, 11/12, 115-130.

1977 «La chemise de l'homme heureux : manipulations narratives sur un conte populaire », avec A. Petitjean, Pratiques, 13, 35-42.

Pratiques du récit, avec A. Petitjean, Cedic, Paris, Nathan.

«Littérature : théorie, enseignement», avec R. Michel et A. Petitjean, Poétique, 30, 156-166.

1978 «Je n'écris pas sur les oiseaux», Pratiques, 21, 35-47.

«L'école et la culture, autrement», avec A. Petitjean, in Ecole, Pouvoir et Démocratie, Metz et Paris, livre collectif Dialectiques et Pratiques, 71-89.

"Lire et écrire en situation scolaire », avec A. Petitjean, Langue Française, 38, 58-73.

1979 «Ce qui se dit quand on parle» in GFEN (dir.), Parler écrire pour de bon à l'école, Paris, Casterman, 21-28.

«Lectures à suivre », Pratiques, 22/23, 37-48.

«L'orthographe sur le terrain », Pratiques, 25, 74-81.

1980 Co-direction de Pratiques, 26, «Ecrire en classe », avec A. Petitjean.

«Ecrits, écriture, école et société », avec A. Petitjean, Pratiques, 26, 323.

« Les maîtres écrivent », Pratiques, 26, 91-93.

"Pour un nouvel enseignement du français », avec A. Petitjean, Pratiques, spécial colloque de Cerisy, 11-23. 
1981 «Pour changer l'écrire », Pratiques, 29, 23-46.

«Les équipes dans l'institution», Pratiques, 31, 12-24.

1982 «Vous voulez jouer au Bac belge?», Pratiques, 33, 61-79.

Direction de Pratiques, 36, « Travailler en projet ».

«Travailler en projet : écrire un roman historique en $5^{\text {ème }}$, Pratiques, $36,38-77$.

« Apprendre autrement à l'école », Pratiques, 36, 5-23.

Pour un nouvel enseignement du français, (co-dir. avec A. Petitjean), Bruxelles, Gembloux, De Boeck-Duculot.

1983 Direction de Pratiques, 40, « La communication».

«De la langue à la communication dans l’école », Pratiques, 40, 3-16.

1984 «L'annotation des copies, variété ou base du dialogue pédagogique », Pratiques, 44, 61-69.

1986 «Où en sommes-nous ? », Repères, 69, 1-2.

"Les pratiques explicatives : points de départ pour une recherche », Repères, 69, INRP, Paris, 3-14.

" Projet collectif et règle du je ", Communiquer ça s'apprend, INRP, Paris, 1986.

1987 « La dynamique des différences dans le travail en projet », Pratiques, 53, 89-107.

Direction, « Un projet didactique, » Repères, 72, INRP, Paris.

«Les conditions de production de l'écrit scolaire », Enjeux, 11, 40-48.

Direction, «Vers une didactique du discours explicatif », Repères, 72, INRP, Paris.

«Ecriture, Littérature, Formation », Cahiers du CRELEF, 25, Université de Franche-Comté, Besançon, 81-104.

1988 Direction de Pratiques, 58, « Les discours explicatifs ».

«Points de vue sur l'explicatif », Pratiques, 58, 3-10.

"L'écriture entre didactique et pédagogie », Etudes de Linguistique appliquée, 71, 7-19.

1989 «Les discours explicatifs : état et perspectives de la recherche », Repères, 77, INRP, Paris, 95-109.

«Savoir écrire, savoir-faire », Pratiques, 61, 3-28.

"Analyse de l'exercice dit "la rédaction" et propositions pour une autre pédagogie de l'écriture », Pour une didactique de l'écriture, CASUM, CRESEF, Metz, 9-47.

1990 La didactique du français, coll. «Que sais-je », PUF, Paris.

Co-direction de Perspectives didactiques en français, Actes du colloque de Cerisy, 1989, coll. " Didactique des textes », Université de Metz.

«Didactique et enseignement du français », in Perspectives didactiques 
en français, Actes du colloque de Cerisy, 1989, coll. «Didactique des textes », Université de Metz, 11-38.

«L'élaboration du texte d'apprenant», Lidil, 3, 98-123.

«L'aide didactique au travail du scripteur», in La production d'écrits de l'école maternelle au collège, Mafpen et CRDP, Dijon, 83-93.

«Texte, discours et didactique de l'explicatif», Recherches, 13, Lille, 16-20.

1993 Direction de Inter-Action, coll. «Didactique des textes », université de Metz.

« L'enjeu didactique de l'interactionnisme », in Inter-Action, coll. « Didactique des textes », université de Metz, 7-39.

1994 «Interaction et apprentissage », Cahiers pédagogiques, 326, 19-22.

«Ecrire le temps en $6^{\text {ème }} / 5^{\text {ème }} »$, Pratiques, 82, 31-70.

Chapitre «Temps et récit», in A. Bentolila et A. Petitjean (dir.), Maîtrise de l'écrit 6ème, manuel de l'élève, Paris, Nathan, 170-181.

Chapitre «Temps et récit», in A. Bentolila et A. Petitjean (dir.), Maîtrise de l'écrit $6^{e m e}$, livre du professeur, Paris, Nathan, 73-78.

1995 « Temps, métalangage et apprentissage », Les métalangages de la classe de français, colloque de la DFLM, Nathan Paris, 1995.

Chapitre «Qu'est-ce qu'écrire? », in A. Bentolila et A. Petitjean (dir.), Maîtrise de l'écrit $5^{\text {ème }}$, manuel de l'élève, Paris, Nathan, 8-30.

Chapitre «Qu'est-ce qu'écrire ? », in A. Bentolila et A. Petitjean (dir.), Maîtrise de l'écrit $5^{\text {ème }}$, livre du professeur, Paris, Nathan, 9-16.

«Interaction. Une problématique à la frontière », in J.-L. Chiss, J. David, Y. Reuter (éds), Didactique du français. Etat d'une discipline, Paris, Nathan pédagogie, 63-78.

1996 Chapitre «Le roman historique », in A. Bentolila et A. Petitjean (dir.), Maîtrise de l'écrit $4^{e ̀ m e}$, manuel de l'élève, Paris, Nathan, 84-104.

Chapitre "Le roman historique », in A. Bentolila et A. Petitjean (dir.), Maîtrise de l'écrit $4^{\text {ème }}$, livre du professeur, Paris, Nathan, 39-44.

" Parler pour apprendre à lire écrire », Les cahiers de Beaumont, Cnefases.

« Sait-on écrire des récits à neuf ans?», Articles, UAB, Espagne.

«Pratiques intégrées de lecture et d'écriture », Pratiques, 90, 61-82.

1997 Chapitre «Continuer une histoire», in A. Bentolila et A. Petitjean (dir.), Maîtrise de l'écrit 3ème, manuel de l'élève, Paris, Nathan, 8-22.

Chapitre «Continuer une histoire», in A. Bentolila et A. Petitjean (dir.), Maîtrise de l'écrit 3ème, livre du professeur, Paris, Nathan, 11-15.

1998 «L'espace didactique et la transposition », Pratiques, 97/98, 171-192.

"L'écriture dans les Instructions Officielles », in A. Petitjean et J.-M. Privat (dir.), 100 ans d'Instructions officielles en français, Actes du colloque de Metz, coll. «Didactique des textes », université de Metz, 215-250. 
«Le Français entre rénovation et reconfiguration », communication au colloque de Tunis, nov. 1998.

1999 Direction de «Interactions et apprentissage », Pratiques, 103/104.

«Les enjeux cognitifs des interactions. Interactions et apprentissage », Pratiques, 103/104, 71-88.

«Conventions, corpus, annexes », avec M. Laparra, Pratiques, 103/104, 223-250.

« Les enjeux didactiques du dyptique langue / discours », communication plénière au séminaire Langue Discours (1999), Université de Madrid.

« De la langue au discours : interactions et didactique », conférence au séminaire Langue Discours (1999), Université de Madrid.

2000 " Des modèles de la didactique aux problèmes de la DFLM », in M. Marquillo Larruy (éd.), Questions d'épistémologie en didactique du français, Les Cahiers Forell, Université de Poitiers, 13-19.

L'oral dans tous ses états, Conférence, publication de l'Académie de Rouen.

Le rôle des interactions langagières dans l'apprentissage dirigé, colloque «Didactique des disciplines et formation des enseignants : approche anthropologique », Marseille, janvier 2000, actes sur le site électronique du colloque, février 2000.

«Le mangeur de têtes », in Le disparu du Pont-Neuf, Paris, Fleurus, 125152.

2001 Des modèles de la didactique aux problèmes de la dflm, in colloque «Questions d'épistémologie en didactique du français », 13-19.

2002 «Pourquoi faut-il oser l'oral ?», Cahiers Pédagogiques, 400.

«Didactique de l'écriture, didactique du français. Vers la cohérence configurationnelle », Pratiques, 115/116, 15-36.

«Enseignement du français », in P. Champy et C. Etévé (dir.), Dictionnaire encyclopédique de l'éducation et de la formation, Paris, Nathan.

2004 «Ecrire de la fiction », l'École des Lettres, 13, 47-55.

«La grammaire au cœur des apprentissages?», in Langue et études de la langue, approches linguistiques et didactiques, C. Vargas (éd.), actes du colloque international de Marseille, 4-6 juin 2003, IUFM d'AixMarseille, Aix en Provence, PUP, 11-23.

"Le français, de la matière à la discipline », Actes du colloque international de l'AIRDF, Le français : discipline singulière, plurielle ou transversale? 26/27/28 août 2004, université Laval, Québec, http://www.colloqueairdf.fse.ulaval.ca

2006 «Entre enseignement et acquisition. Problèmes didactiques en apprentissage du langage ", Mélanges CRAPEL, 29, 13-28. 